\title{
Acknowledgement of reviewers for 2020
}

๑) Springer Nature B.V. 2021

Please join us in thanking all those scientists and experts in the various fields represented in Natural Hazards for devoting time and effort to review the papers that we have been sending them. The Editors-in-Chief and Publisher acknowledge these colleagues for their excellent reviews of papers in 2020. 